\title{
Derivation of Transformation and One-Way Speed of Light in Kinematics of Special Theory of Ether
}

\author{
Karol Szostek ${ }^{1}$, Roman Szostek ${ }^{2}$ \\ ${ }^{1}$ Department of Thermodynamics and Fluid Mechanics, Rzeszow University of Technology, Rzeszow, Poland \\ ${ }^{2}$ Department of Quantitative Methods, Rzeszow University of Technology, Rzeszow, Poland
}

Email address:

kszostek@prz.edu.pl (K. Szostek), rszostek@prz.edu.pl (R. Szostek)

To cite this article:

Karol Szostek, Roman Szostek. Derivation of Transformation and One-Way Speed of Light in Kinematics of Special Theory of Ether. American Journal of Modern Physics. Vol. 6, No. 6, 2017, pp. 140-147. doi: 10.11648/j.ajmp.20170606.15

Received: September 28, 2017; Accepted: October 10, 2017; Published: October 31, 2017

\begin{abstract}
At present, it is believed that the Special Theory of Relativity (STR) is the only theory explaining the MichelsonMorley and Kennedy-Thorndike experiments. This article proved that another theory in accordance with these experiments is possible. In this article, we derive the new theory of kinematics of bodies from the universal frame of reference (UFR, ether), which we called the Special Theory of Ether (STE). The article explains why Michelson-Morley and Kennedy-Thorndike experiments could not detect the universal frame of reference. In article, a different transformation of time and position than the Lorentz transformation is derived on the basis of the geometric analysis of the Michelson-Morley and Kennedy-Thorndike experiments. The formula for summation of speeds for absolute speed has been derived. Based on the derived transformation, we derive the formula for the velocity of light in vacuum measured in any inertial reference system. The entire article contains only original research conducted by its authors.
\end{abstract}

Keywords: Kinematics of Bodies, Universal Frame of Reference, Coordinate and Time Transformation, One-Way Speed of Light

\section{Introduction}

The article an explanation the results of the MichelsonMorley [1] and Kennedy-Thorndike [2] experiments, assuming the existence of the universal frame of reference (UFR), in which the velocity of light is constant, is presented. In inertial frames of reference moving in the UFR, the oneway velocity of light may be different. The transformations from the inertial system to the UFR and from the UFR to the inertial system was derived by the geometric method.

The velocity of light in one direction has never been accurately measured. In all accurate laboratory experiments, as in the Michelson-Morley experiment, only the average velocity of light, travelling on a closed trajectory, was measured. In these experiments, light always comes back to the source point. Therefore, the assumption about the constant velocity of light (instantaneous velocity) adopted in the Special Theory of Relativity is not experimentally justified. The derivation presented in this article is based on the assumption resulting from these experiments, that is for every observer, the average velocity of light travelling the way to and back is constant.

The transformation «UFR- inertial system» (27)-(28) derived in this article by the geometric method was already derived in articles [3] and [4] by other method. In article [3] the author obtained this transformation from the Lorentz transformation thanks to the synchronization of clocks in inertial frames by the external method. The transformation obtained in the work [3] is the Lorentz transformation differently written down after a change in the manner of time measurement in the inertial frame of reference, this is why the properties of the Special Theory of Relativity were attributed to this transformation. The transformation (27)(28) has a different physical meaning than the Lorentz transformation, because according to the theory outlined in this article, it is possible to determine the speed with respect to a universal frame of reference by local measurement. So the universal reference system is real, and this is not a freely chosen inertial system. 


\section{The Assumptions}

In the presented analysis of the Michelson-Morley and Kennedy-Thorndike experiments, the following assumptions are adopted:

I. There is a universal frame of reference (UFR) with respect to which the velocity of light in vacuum is the same in every direction.

II. The average velocity of light on its way to and back is for every observer independent of the direction of light propagation. This results from the Michelson-Morley experiment.

III. The average velocity of light on its way to and back does not depend on the velocity of the observer in relation to the UFR. This results from the KennedyThorndike experiment.

IV.In the direction perpendicular to the direction of the velocity of the body, moving in relation to the UFR, there is no contraction or elongation of its length.

V. The transformation «UFR-inertial system» is linear.

The transformation derivation presented in this article differs from the derivation by the geometric method of the Lorentz transformation which is the basis for the STR. In STR in the derivation of the Lorentz transformation, it is assumed that the reverse transformation has the same form as the original transformation. Such an assumption stems from the belief that all inertial frames are equivalent. In the derivation presented in this article, we do not assume what form the reverse transformation has.

Assumptions concerning the velocity of light adopted in this article are also weaker than those adopted in the STR. In the STR, it is assumed that the velocity of light is absolutely constant, despite the fact that it has not been proven by any experiment. In this article, the assumption resulting from experiments is adopted, i.e. the average velocity of light on the way to the mirror and back is constant (assumption II and III). In the presented considerations, the velocity of light by assumption is constant only in one highlighted frame of reference - the UFR (assumption I).

Assumptions IV and V are identical to those on which the STR is based.

In works [7] and [8], identical transformations were derived as in this article, but with the adopted additional assumption. For this, it was necessary to conduct the full analysis of the Michelson-Morley experiment in which also the second stream of light, parallel to velocity $v$, is taken into account. In that case, only one stream of light was analyzed.

\section{Time and Way of the Light Flow in the UFR}

Let us consider inertial system $U^{\prime}$, which moves in relation to system $U$ related with the UFR at velocity $v$ (Figure 1 ). In system $U^{\prime}$, there is a mirror at distance $D^{\prime}$ from the beginning of the system. Light in the system $U$ moves at constant velocity $c$. From system $U^{\prime}$, from point $x^{\prime}=0$ in time $t=0$, a stream of light was sent in the direction of the mirror. Having reached the mirror, the reflected light moves in the system $U$ in the opposite direction at velocity with the negative value $c$.

We assume the following symbols for the observer from the system $U: t_{1}$ is the time of the light flow to the mirror, $t_{2}$ is the time of the light return to the starting point. $L_{1}$ and $L_{2}$ are ways which were travelled by light in the system $U$ in one direction and in another.

When light moves in the direction of the mirror, then the mirror runs away from it at velocity $v$. When light comes back to point $x^{\prime}=0$ after the reflection from the mirror, then this point runs towards it at velocity $v$. For an observer from system $U$, distance $D^{\prime}$ parallel to velocity vector $v$ is seen as $D$. We obtain

$$
\begin{gathered}
L_{1}=D+v \cdot t_{1}, \quad L_{2}=D-v \cdot t_{2} \\
t_{1}=\frac{L_{1}}{c}=\frac{D+v \cdot t_{1}}{c}, \quad t_{2}=\frac{L_{2}}{c}=\frac{D-v \cdot t_{2}}{c}
\end{gathered}
$$

a)

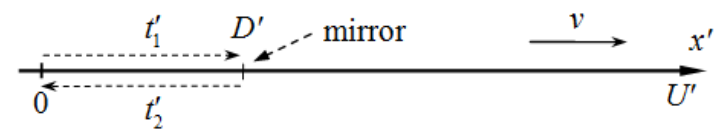

b)

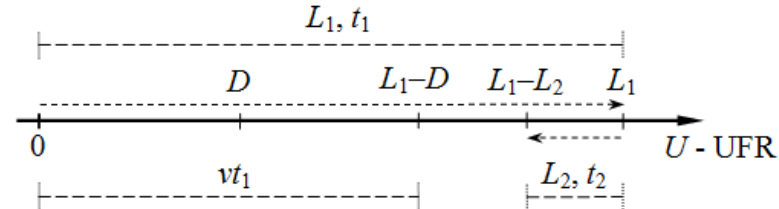

Figure 1. The Time and the Flow Path of Light to the Mirror and Back.

a) the way of light seen from the inertial system $U^{\prime}$,

$b$ ) the way of light seen from the UFR.

Dependencies (2) should be solved due to $t_{1}$ and $t_{2}$. We then obtain time and way of flow in the UFR

$$
\begin{gathered}
t_{1}=\frac{D}{c-v}, \quad t_{2}=\frac{D}{c+v} \\
L_{1}=c \cdot t_{1}=D \frac{c}{c-v}, \quad L_{2}=c \cdot t_{2}=D \frac{c}{c+v}
\end{gathered}
$$

\section{The Geometrical Derivation of the Transformation}

We analyze the results of the Michelson-Morley experiment, as shown in Figure 2. The inertial system $U^{\prime}$ move sat a relative velocity $v$ to the inertial system $U$, associated with the UFR, parallel to the axis $x$. Axes $x$ and $x^{\prime}$ lie on one straight line.

At the moment when origins of systems overlap, clocks in both systems are synchronized. Clocks in system $U$ related to the UFR are synchronized by the internal method [3]. Clocks in system $U^{\prime}$ are synchronized by the external method in such a manner that if the clock of system $U$ indicates time $t=0$, then the clock of system $U^{\prime}$ next to it is also reset, that is $t^{\prime}=0$.

In the system $U^{\prime}$, an experiment measuring the velocity of 
light in vacuum perpendicular and parallel to the direction of movement of the system $U^{\prime}$ in relation to the UFR was conducted. In each of these directions, light travels to the mirror and back. Figure 2 presents in part $a$ ) the flow path of light seen by the observer from the system $U^{\prime}$, while in part $b)$ the path seen by the observer from the system $U$.

In system $U$ light has always constant velocity $c$ (assumption I). Considerations concern the flow of light in vacuum.

In accordance with conclusions resulting from the Michelson-Morley experiment it has been assumed that the average velocity of light $c_{p}$ on the way to the mirror and back in system $U^{\prime}$ is the same in every direction, in particular in the parallel direction to the axis $y^{\prime}$ (assumption II). It has also been assumed that the average velocity of light $c_{p}$ on the way to the mirror and back does not depend on the velocity of an observer in relation to the UFR (assumption III).
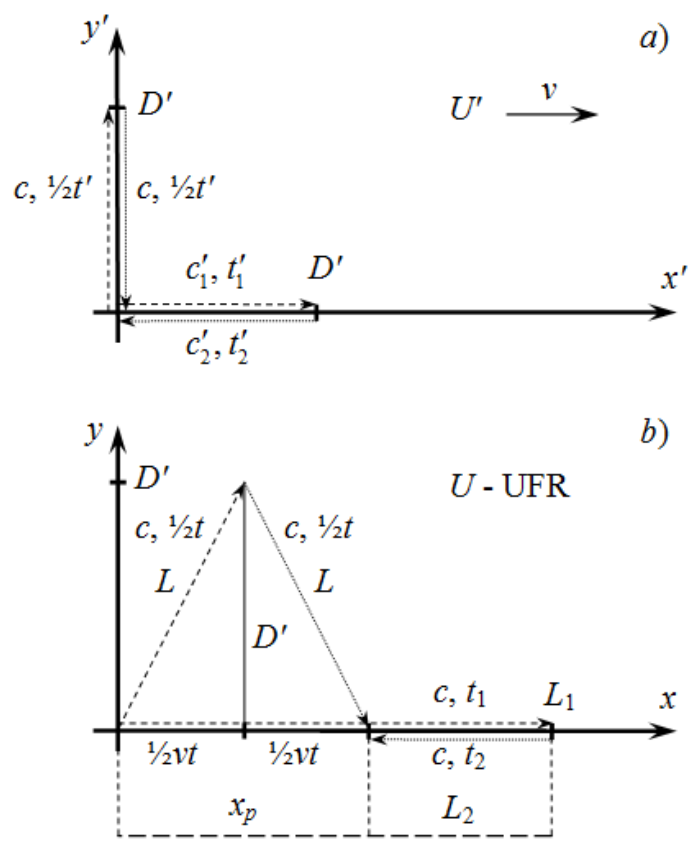

Figure 2. Paths of Two Streams of Light.

a) seen by an observer from the system $U^{\prime}$,

$b$ ) seen by an observer from the system $U$ (UFR).

From assumption II and III it follows that the average velocity of light $c_{p}$ in the inertial frame of reference is the same as the velocity of light $c$ in the system $U$. If we allow that the average velocity of light $c_{p}$ in the system $U^{\prime}$ is a function of the velocity of light $c$ in the system $U$ dependent on the velocity $v$, we can write

$$
c_{p}=f(v) c
$$

From assumption III the average velocity of light is the same for different velocities of the Earth relative to the UFR, so $f\left(v_{1}\right)=f\left(v_{2}\right)$. Since $f(0)=1$, therefore $f(v)=1$ for every velocity $v$. It follows that $c=c_{p}$.

The mirrors are associated with the system $U^{\prime}$ and placed at distance $D^{\prime}$ from the origin. One mirror is located on the axis $x^{\prime}$, the second one on the axis $y^{\prime}$. We assume that the distance $D^{\prime}$, which is perpendicular to the velocity $v$ is the same for observers from both systems (assumption IV). Therefore, in Figure 2, there is the same length $D^{\prime}$ in part $a$ ) and part $b$ ).

The flow time of light in the system $U$, along the axis $x$, in the direction to the mirror is marked as $t_{1}$. The flow time back is marked as $t_{2}$.

The flow time of light in the system $U^{\prime}$, along the axis $x^{\prime}$, in the direction to the mirror is marked as $t_{1}^{\prime}$. The flow time back to the source is marked $t_{2}^{\prime}$.

Total time is marked respectively as $t$ and $t^{\prime}\left(t=t_{1}+t_{2}\right.$ and $t^{\prime}$ $\left.=t_{1}^{\prime}+t_{2}^{\prime}\right)$.

The light stream, moving parallel to the axis $y^{\prime}$, from the point of view of the system $U$ moves along the arms of an isosceles triangle of side length $L$. Since the velocity of light is constant in the system $U$, therefore, the time of movement along both arms is the same and is equal to $t / 2$.

In the system $U$, the light stream parallel to the axis $x$, in the direction of the mirror overcomes distance $L_{1}$ during time $t_{1}$. On the way back, it travels distance $L_{2}$ during time $t_{2}$. These distances are different due to the movement of the mirror and the source point of light in the UFR.

In the experiment, both light streams come back to the source point at the same time, both in system $U$ and system $U^{\prime}$. It results from assumption II and from the mirrors setting at the same distance from the point of light emission.

For an observer of $U^{\prime}$ and $U$, the velocity of light can be written as

$$
\frac{2 D^{\prime}}{t_{1}^{\prime}+t_{2}^{\prime}}=\frac{2 D^{\prime}}{t^{\prime}}=c=\frac{2 L}{t}=\frac{L_{1}+L_{2}}{t_{1}+t_{2}}
$$

From equation (6) light paths $L$ and $D^{\prime}$ as a function of the velocity of light $c$ and the light flow times $t, t^{\prime}$ respectively in the systems $U$ and $U^{\prime}$ can be determined

$$
L=\frac{c t}{2} ; \quad D^{\prime}=\frac{c t^{\prime}}{2}
$$

The velocity of the system $U^{\prime}$ relative to the absolute frame of reference $U$, i.e. the UFR is marked by $v$. Since $x_{p}$ is the path that the system $U^{\prime}$ travelled in time $t$, of the light flow, we have

$$
v=\frac{x_{p}}{t} ; \quad x_{p}=v t
$$

Using the geometry of Figure 2, the length $L$ can be expressed as

$$
L=\sqrt{\left(x_{p} / 2\right)^{2}+D^{\prime 2}}=\sqrt{(v t / 2)^{2}+D^{\prime 2}}
$$

Having squared equation (9) and taken (7) into account, we obtain

$$
(c t / 2)^{2}=(v t / 2)^{2}+\left(c t^{\prime} / 2\right)^{2}
$$


After arranging we obtain

$$
\begin{gathered}
t^{2}\left(c^{2}-v^{2}\right)=\left(c t^{\prime}\right)^{2} \\
t=t^{\prime} \frac{1}{\sqrt{1-(v / c)^{2}}} \text { for } x^{\prime}=0
\end{gathered}
$$

The above relation describes only times $t$ and $t^{\prime}$ that involve the full light flow to the mirror and back. It should be noted that these are times measured in point $x^{\prime}=0$. However, if we assume that the length $D^{\prime}$ can be chosen so that time flow of light is any time, so the relationship (12) is true for any time.

Length $D^{\prime}$ associated with the system $U^{\prime}$ that is parallel to the axis $x$, and is seen from the system $U$ as $D$. If light flows in the absolute frame of reference $U$ to the mirror, is chasing the mirror, which is away from it at length $D$. After reflection, light returns to the source point, which runs against him. Using equations (4), we obtain the equations for light flow paths in both directions along the axis $x^{\prime}$ in the system $U$

$$
L_{1}=c t_{1}=D \frac{c}{c-v} ; \quad L_{2}=c t_{2}=D \frac{c}{c+v}
$$

From equations (13) the sum and difference in length the $L_{1}$ and $L_{2}$, which light travelled in the system $U$, can be determined

$$
\begin{aligned}
& L_{1}+L_{2}=D \frac{c}{c-v}+D \frac{c}{c+v}=2 D \frac{1}{1-(v / c)^{2}} \\
& L_{1}-L_{2}=D \frac{c}{c-v}+D \frac{c}{c+v}=2 D \frac{v}{c} \cdot \frac{1}{1-(v / c)^{2}}
\end{aligned}
$$

From the second equation, the distance that the system $U^{\prime}$ travelled in half of the light flow time $t / 2$ can be determined, so we have

$$
\frac{x_{p}}{2}=\frac{v t}{2}=\frac{L_{1}-L_{2}}{2}=D \frac{v}{c} \cdot \frac{1}{1-(v / c)^{2}}
$$

Since it was assumed that in the system $U$ the velocity of light $c$ is constant, therefore both distances, which are travelled by light $2 L$ and $L_{1}+L_{2}$ are the same

$$
2 L=L_{1}+L_{2}
$$

After substituting (9) and the first equation (14) we obtain

$$
2 \sqrt{(v t / 2)^{2}+D^{\prime 2}}=2 D \frac{1}{1-(v / c)^{2}}
$$

After reducing by two, raising to the square and taking (15) into account we can write

$$
\left(D \frac{v}{c} \cdot \frac{1}{1-(v / c)^{2}}\right)^{2}+D^{\prime 2}=D^{2}\left(\frac{1}{1-(v / c)^{2}}\right)^{2}
$$

From equation (18) a dependence for the length contraction can be determined

$$
\begin{gathered}
D^{\prime 2}=D^{2}\left(\frac{1}{1-(v / c)^{2}}\right)^{2}\left(1-(v / c)^{2}\right) \\
D^{\prime}=D\left(\frac{1}{1-(v / c)^{2}}\right) \sqrt{1-(v / c)^{2}}=D \frac{1}{\sqrt{1-(v / c)^{2}}} \\
D=D^{\prime} \sqrt{1-(v / c)^{2}}
\end{gathered}
$$

Lengths $D$ and $D^{\prime}$ which are distances between mirrors and the point of light emission occur in the above dependence. Since length $D^{\prime}$ can be selected on a voluntary basis; therefore, dependence (20) is true for any value of $D^{\prime}$.

Having introduced (12) to (8), we have

$$
x_{p}=v t^{\prime} \frac{1}{\sqrt{1-(v / c)^{2}}} \text { for } x^{\prime}=0
$$

We assume that the transformation from the inertial system $U^{\prime}$ to the system $U$ is linear (assumption V). If linear factors dependent on $x^{\prime}$ are added to the transformation of time and position (12), (21), transformations with unknown coefficients $a, b$ can be obtained

$$
\begin{aligned}
& t=t^{\prime} \frac{1}{\sqrt{1-(v / c)^{2}}}+a x^{\prime} \\
& x=v t^{\prime} \frac{1}{\sqrt{1-(v / c)^{2}}}+b x^{\prime}
\end{aligned}
$$

Transformation (22) should be valid for any time and position. In a particular case, it is valid at the moment of clocks' synchronization, that is when $t=t^{\prime}=0$ for the point with coordinates $D^{\prime}$ in system $U^{\prime}$. In this connection, we introduce $t=t^{\prime}=0, x^{\prime}=D^{\prime}$ and $x=D$ into (22). Having taken (20) into account, we obtain

$$
\begin{aligned}
& 0=a D^{\prime} \\
& \sqrt{1-(v / c)^{2}} D^{\prime}=b D^{\prime}
\end{aligned}
$$

We obtain coefficients $a$ and $b$

$$
\begin{aligned}
& a=0 \\
& b=\sqrt{1-(v / c)^{2}}
\end{aligned}
$$

Finally, the transformation from any inertial system $U^{\prime}$ to the system $U$, associated with the UFR takes the form

$$
\begin{gathered}
t=\frac{1}{\sqrt{1-(v / c)^{2}}} t^{\prime} \\
x=\frac{1}{\sqrt{1-(v / c)^{2}}} v t^{\prime}+\sqrt{1-(v / c)^{2}} \cdot x^{\prime}
\end{gathered}
$$


After transformations of the above equations, we obtain the inverse transformation, that is the transformation from the system $U$, associated with the UFR to the inertial system $U^{\prime}$

$$
\begin{gathered}
t^{\prime}=\sqrt{1-(v / c)^{2}} \cdot t \\
x^{\prime}=\frac{1}{\sqrt{1-(v / c)^{2}}}(-v t+x)
\end{gathered}
$$

Due to the assumption IV also occurs

$$
y^{\prime}=y \quad \text { and } \quad z^{\prime}=z
$$

The velocity $v$ is the velocity of the inertial system relative to the universal reference system.

\section{The Transformation of Velocity}

Axes of the inertial system $U^{\prime}$ and the system $U$ connected with the ether were determined in such a way that they were parallel to each other (Figure 3 ). The inertial system moves at the velocity $v$ in parallel to the axis $x$ and $x^{\prime}$.
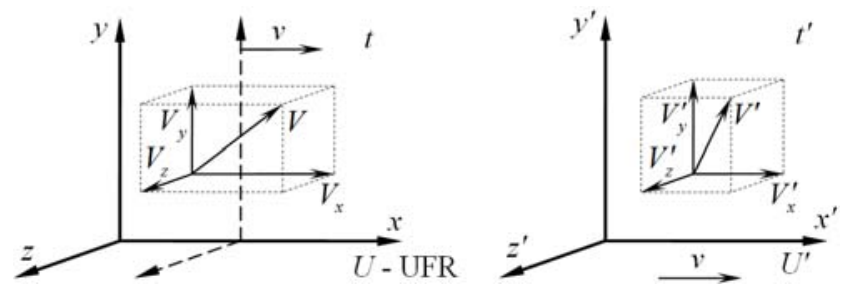

Figure 3. The Movement Seen from the Ether and the Inertial System.

Differentials from the transformation (27)-(29) have the form

$$
\left\{\begin{array}{l}
d t^{\prime}=\sqrt{1-(v / c)^{2}} d t \\
d x^{\prime}=\frac{1}{\sqrt{1-(v / c)^{2}}}(-v d t+d x) \\
d y^{\prime}=d y \\
d z^{\prime}=d z
\end{array}\right.
$$

A moving body is observed from the ether $U$ and the inertial system $U^{\prime}$. In the ether, it moves at the velocity $V$, while in the inertial system, it moves at the velocity $V^{\prime}$. Components of these velocities are presented in Figure 3.

The velocity of the body in the system of the ether $U$ can be written in the form

$$
V_{x}=\frac{d x}{d t}, \quad V_{y}=\frac{d y}{d t}, \quad V_{z}=\frac{d z}{d t}
$$

The velocity of the body in the inertial system $U^{\prime}$ can be written in the form

$$
V_{x}^{\prime}=\frac{d x^{\prime}}{d t^{\prime}}, \quad V_{y}^{\prime}=\frac{d y^{\prime}}{d t^{\prime}}, \quad V_{z}^{\prime}=\frac{d z^{\prime}}{d t^{\prime}}
$$

We introduce differentials (32) into equations (30). We obtain

$$
\left\{\begin{array}{l}
V_{x}^{\prime}=\frac{\frac{1}{\sqrt{1-(v / c)^{2}}}(-v d t+d x)}{\sqrt{1-(v / c)^{2}} d t} \\
V_{y}^{\prime}=\frac{d y}{\sqrt{1-(v / c)^{2}} d t} \\
V_{z}^{\prime}=\frac{d z}{\sqrt{1-(v / c)^{2}} d t}
\end{array}\right.
$$

That is

$$
\left\{\begin{array}{l}
V_{x}^{\prime}=\frac{-v}{1-(v / c)^{2}}+\frac{1}{1-(v / c)^{2}} \frac{d x}{d t} \\
V_{y}^{\prime}=\frac{1}{\sqrt{1-(v / c)^{2}}} \frac{d y}{d t} \\
V_{z}^{\prime}=\frac{1}{\sqrt{1-(v / c)^{2}}} \frac{d z}{d t}
\end{array}\right.
$$

Based on (31), we obtain the searched transformation of velocity

$$
\left\{\begin{array}{l}
V_{x}^{\prime}=\frac{V_{x}-v}{1-(v / c)^{2}} \\
V_{y}^{\prime}=\frac{V_{y}}{\sqrt{1-(v / c)^{2}}} \\
V_{z}^{\prime}=\frac{V_{z}}{\sqrt{1-(v / c)^{2}}}
\end{array}\right.
$$

Transformation (35) expresses the relative velocity $V^{\prime}$ from the absolute velocities $V$ and $v$. From the first equation of this transformation can obtain a formula for summing the parallel velocities in the form

$$
V_{x}=v+V_{x}^{\prime}\left(1-(v / c)^{2}\right)
$$

and formula for relative velocity in form

$$
V_{x}^{\prime}=\frac{V_{x}-v}{1-(v / c)^{2}}
$$

\section{The Velocity of Light in Vacuum for a Moving Observer}

Generally, the light flow occurs along paths presented in Figure 4. Axes of coordinate systems are set in such a way that

$$
c_{z}=c_{z}^{\prime}=0
$$




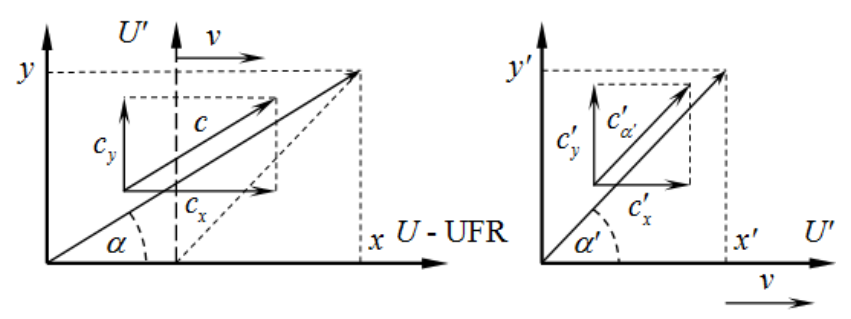

Figure 4. The Light Flow at Any Angle.

In accordance with the Figure based on the Pythagorean theorem, we obtain

$$
\begin{aligned}
& c_{\alpha^{\prime}}^{\prime 2}=c_{x}^{\prime 2}+c_{y}^{\prime 2} \\
& c^{2}=c_{x}^{2}+c_{y}^{2}
\end{aligned}
$$

The following also occurs

$$
\cos \alpha^{\prime}=\frac{c_{x}^{\prime}}{c_{\alpha^{\prime}}^{\prime}}
$$

When $V_{x}=c_{x}$ and $V_{x}^{\prime}=c_{x}^{\prime}$, then in accordance with (35) the following occurs

$$
\begin{aligned}
& c_{x}^{\prime}=\frac{c_{x}-v}{1-(v / c)^{2}} \\
& c_{y}^{\prime}=\frac{c_{y}}{\sqrt{1-(v / c)^{2}}}
\end{aligned}
$$

\subsection{The First Dependence for the Velocity of Light}

Having introduced dependencies (42) and (43) into (39), we obtain

$$
\begin{gathered}
c_{\alpha^{\prime}}^{\prime 2}=\left(\frac{c_{x}-v}{1-(v / c)^{2}}\right)^{2}+\left(\frac{c_{y}}{\sqrt{1-(v / c)^{2}}}\right)^{2} \\
c_{\alpha^{\prime}}^{\prime 2}=c^{4} \frac{\left(c_{x}-v\right)^{2}}{\left(c^{2}-v^{2}\right)^{2}}+c^{2} \frac{c_{y}^{2}}{c^{2}-v^{2}} \\
c_{\alpha^{\prime}}^{\prime 2}=\frac{c^{2}}{\left(c^{2}-v^{2}\right)^{2}}\left[c^{2}\left(c_{x}-v\right)^{2}+\left(c^{2}-v^{2}\right) c_{y}^{2}\right]
\end{gathered}
$$

Having taken (40) into account, we obtain

$$
\begin{gathered}
c_{\alpha^{\prime}}^{\prime 2}=\frac{c^{2}}{\left(c^{2}-v^{2}\right)^{2}}\left[c^{2}\left(c_{x}^{2}-2 v c_{x}+v^{2}\right)+\left(c^{2}-v^{2}\right)\left(c^{2}-c_{x}^{2}\right)\right] \\
c_{\alpha^{\prime}}^{\prime 2}=\frac{c^{2}}{\left(c^{2}-v^{2}\right)^{2}}\left(c^{2} c_{x}^{2}-2 v c^{2} c_{x}+v^{2} c^{2}+c^{4}-c^{2} c_{x}^{2}-v^{2} c^{2}+v^{2} c_{x}^{2}\right) \\
c_{\alpha^{\prime}}^{\prime 2}=\frac{c^{2}}{\left(c^{2}-v^{2}\right)^{2}}\left(-2 v c^{2} c_{x}+c^{4}+v^{2} c_{x}^{2}\right)
\end{gathered}
$$

$$
c_{\alpha^{\prime}}^{\prime 2}=\frac{c^{2}}{\left(c^{2}-v^{2}\right)^{2}}\left(c^{2}-v c_{x}\right)^{2}
$$

On this basis, we obtain the first dependence for the velocity of light in the inertial system expressed from $c_{x}$

$$
c_{\alpha^{\prime}}^{\prime}=\frac{c}{c^{2}-v^{2}}\left(c^{2}-v c_{x}\right)
$$

\subsection{The Second Dependence for the Velocity of Light}

Based on (42) we obtain

$$
c_{x}=v+\left(1-(v / c)^{2}\right) c_{x}^{\prime}=v+\frac{c^{2}-v^{2}}{c^{2}} c_{x}^{\prime}
$$

After introducing it into (51), we obtain

$$
\begin{gathered}
c_{\alpha}^{\prime}=\frac{c}{c^{2}-v^{2}}\left[c^{2}-v\left(v+\frac{c^{2}-v^{2}}{c^{2}} c_{x}^{\prime}\right)\right] \\
c_{\alpha^{\prime}}^{\prime}=\frac{c}{c^{2}-v^{2}}\left[c^{2}-v^{2}-v \frac{c^{2}-v^{2}}{c^{2}} c_{x}^{\prime}\right] \\
c_{\alpha^{\prime}}^{\prime}=c-\frac{v c_{x}^{\prime}}{c}
\end{gathered}
$$

On this basis we obtain the second dependence for the velocity of light in the inertial system, expressed from $c_{x}^{\prime}$

$$
c_{\alpha^{\prime}}^{\prime}=\frac{c^{2}-v c_{x}^{\prime}}{c}
$$

\subsection{The Third Dependence for the Velocity of Light}

Based on (56) we obtain

$$
\begin{gathered}
c c_{\alpha^{\prime}}^{\prime}=c^{2}-v c_{x}^{\prime} \\
c c_{\alpha^{\prime}}^{\prime}+v c_{x}^{\prime}=c^{2} \\
1=\frac{c^{2}}{c c_{\alpha^{\prime}}^{\prime}+v c_{x}^{\prime}} \\
c_{\alpha^{\prime}}^{\prime}=\frac{c^{2} c_{\alpha^{\prime}}^{\prime}}{c c_{\alpha^{\prime}}^{\prime}+v c_{x}^{\prime}} \\
c_{\alpha^{\prime}}^{\prime}=\frac{c^{2}}{c+v \frac{c_{x}^{\prime}}{c_{\alpha^{\prime}}^{\prime}}}
\end{gathered}
$$

From this equation based on (41) we obtain the third dependence for the velocity of light in the inertial system, expressed from $\alpha^{\prime}$ (Figure 5) 


$$
c_{\alpha^{\prime}}^{\prime}=\frac{c^{2}}{c+v \cos \alpha^{\prime}}
$$

This formula is identical to formula (377) derived by the geometric method in the work [6].

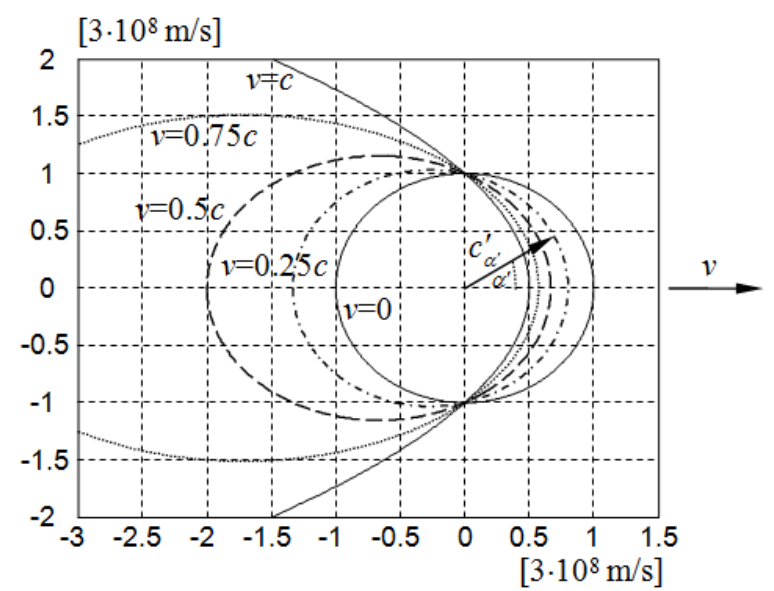

Figure 5. The Velocity of Light $c_{\alpha^{\prime}}^{\prime}$ in the Inertial System for $v=0,0.25 c, 0.5 c$, $0.75 c, c$.

We will now determine the average velocity of light which in any inertial system travels the path with the length $L^{\prime}$, is reflected from the mirror and returns along the same path to the source point (Figure 6). If $t_{1}^{\prime}$ is the time needed for light to travel the path $L^{\prime}$ in one direction, while $t_{2}^{\prime}$ is the time needed for light to travel the same path in the other direction, then the average velocity of light along the path back and forth is equal to

$$
\begin{gathered}
c_{s r}^{\prime}=\frac{2 L^{\prime}}{t_{1}^{\prime}+t_{2}^{\prime}}=\frac{\frac{2 L^{\prime}}{\frac{L^{\prime}}{c+v \cos \alpha^{\prime}}}+\frac{\frac{c^{\prime}}{c+c \cos \left(\pi-\alpha^{\prime}\right)}}{c_{s r}^{2}}}{\frac{c+v \cos \alpha^{\prime}}{c^{2}}+\frac{c-v \cos \alpha^{\prime}}{c^{2}}}=\frac{2}{\frac{2 c}{c^{2}}}=c
\end{gathered}
$$

It follows that the average speed of light is constant and equal to the one-way speed of light $c$ seen from the ether. This average velocity does not depend on the angle $\alpha^{\prime}$ nor the velocity $v$. For this reason, the rotation of the interferometer in Michelson-Morley and Kennedy-Thorndike experiments does not influence interference fringes. Therefore, these experiments could not detect the ether.

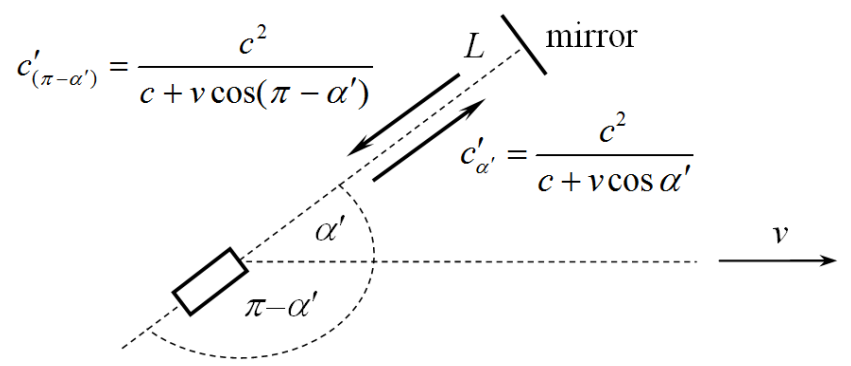

Figure 6. The Velocity of Light in the Michelson-Morley Experiment.
In work [6] a formula for the velocity of light running in any direction in a material medium motionless in relation to the observer, more general than formula (62), is derived by means of the geometric method. It has the form of $\left(c_{s}\right.$ is the average velocity of light traveling the path to and back in this material medium)

$$
c_{s \alpha^{\prime}}^{\prime}=\frac{c^{2} c_{s}}{c^{2}+c_{s} v \cos \alpha^{\prime}}
$$

\section{Conclusion}

It follows from the conducted analysis that the explanation of the results of the Michelson-Morley experiment on the basis of the universal frame of reference is possible. Stating that the Michelson-Morley experiment proved that the velocity of light is absolutely constant is untrue. Stating that the Michelson-Morley experiment proved that there is no universal frame of reference in which light propagates and moves at a constant velocity is also untrue. From the derived transformations (25)-(26) and (27)-(28) it follows that the measurement of the velocity of light in vacuum by means of the previously applied methods will always give the average value equal to $c$. This happens despite the fact that for the moving observer the velocity of light has a different value in different directions. The average velocity of light is always constant and independent of the velocity of the inertial frame of reference. Due to this property of the velocity of light, the Michelson-Morley and Kennedy-Thorndike experiments could not detect the universal frame of reference.

Admitting that the velocity of light may depend on the direction of its emission does not differentiate any direction in space. The velocity of light which is measured by the moving observer is significant here. It is the velocity at which the observer moves in relation to the universal frame of reference that differentiates the characteristic direction in space, but only for this observer. For the observer motionless in relation to the universal frame of reference, the velocity of light is always constant and does not depend on the direction of its emission. If the observer moves in relation to the universal frame of reference, then from his perspective space is not symmetrical. The case of this observer will be similar to the case of the observer moving on water and measuring the velocity of the wave on water. Despite that the wave propagates on water at the constant velocity in every direction, from the perspective of the observer moving on water, the velocity of the wave will be different in different directions.

In works [6]-[10], a new physical theory of kinematics and dynamics of bodies based on the transformation determined here, called by the authors the Special Theory of Ether, was derived. In work [10] it has been shown that it is possible to weaken the assumption IV and derive a more general form of transformation (25)-(26) and (27)-(28). Thus many kinematics can be derived in accordance with the Michelson-Morley and Kennedy-Thorndike experiments. In the work [6] has been shown that within each such kinematics can derive infinitely many dynamics. In order to derive dynamics, it is necessary 
to adopt the additional assumption, which will allow for introduction into theory of the concepts of mass, kinetic energy, and momentum.

Based on this kinematics can naturally explain the anisotropy of the microwave background radiation, which was discussed at work [5]. This allows determine the speed at which the solar system is moving relative to a universal reference system, that is $369.3 \mathrm{~km} / \mathrm{s}$. This has been shown in [8] and [10].

The Michelson-Morley experiment and KennedyThorndike experiment were conducted many times by different teams. Each of the experiments only confirmed that the average velocity of light is constant. Therefore, assumptions, on which the presented derivation is based, are experimentally justified.

\section{References}

[1] Michelson Albert A., Morley Edward W., On the relative motion of the earth and the luminiferous ether. Am. J. Sci. 34, 333-345, 1887.

[2] Kennedy Roy J., Thorndike Edward M., Experimental Establishment of the Relativity of Time. Physical Review, 42 (3), 400-418, 1932.

[3] Mansouri Reza, Sexl Roman U., A Test Theory of Special Relativity: I. Simultaneity and Clock Synchronization. General Relativity and Gravitation, Vol. 8, No. 7, 1977, 497-513.

[4] Tangherlini Frank R., The Velocity of Light in Uniformly Moving Frame. A Dissertation. Stanford University, 1958 (reprint in The Abraham Zelmanov Journal, Vol. 2, 2009, ISSN 1654-9163).

[5] Smoot George F., Nobel Lecture: Cosmic microwave background radiation anisotropies: Their discovery and utilization (in English). Reviews of Modern Physics, Volume
79, 1349-1379, 2007. Smoot George F., Anizotropie kosmicznego mikrofalowego promieniowania tta: ich odkrycie $i$ wykorzystanie (in Polish). Postępy Fizyki, Tom 59, Zeszyt 2, 52-79, 2008. Смут Джордж Ф., Анизотропия реликтового излучения: открытие и научное значение (in Russian). Успехи Физических Наук, Том 177, № 12, 1294-1317, 2007.

[6] Szostek Karol, Szostek Roman, Special Theory of Ether (in English), Publishing house Amelia, Rzeszow, Poland, 2015, www.ste.com.pl, ISBN 978-83-63359-81-2. Szostek Karol, Szostek Roman, Szczególna Teoria Eteru (in Polish), Wydawnictwo Amelia, Rzeszów, Polska, 2015, www.ste.com.pl, ISBN 978-83-63359-77-5.

[7] Szostek Karol, Szostek Roman, The Geometric Derivation of the Transformation of Time and Position Coordinates in STE, IOSR Journal of Applied Physics (IOSR-JAP), Volume 8, Issue 4, Version III, 22-30, 2016, ISSN 2278-4861, doi:10.9790/4861-0804032230.

[8] Szostek Karol, Szostek Roman, Выделенная в космологии система отсчета и возможная модификаиия преобразований Лоренща (in Russian: The preferential reference system in cosmology and the possible modification of Lorentz transformation). Ученые Записки Физического Факультета МГУ (Memoirs of the Faculty of Physics Lomonosov Moscow State University), № 2, 2017, 172102, ISSN 2307-9665.

[9] Szostek Karol, Szostek Roman, The explanation of the Michelson-Morley experiment results by means of the theory of ether (in English). viXra 2016, www.vixra.org/abs/1612.0019. Szostek Karol, Szostek Roman, Wyjaśnienie wyników eksperymentu MichelsonaMorleya przy pomocy teorii z eterem (in Polish). viXra 2017, www.vixra.org/abs/1704.0302.

[10] Szostek Karol, Szostek Roman, The Derivation of the General Form of Kinematics with the Universal Reference System (in English). viXra 2017, www.vixra.org/abs/1704.0105. Szostek Karol, Szostek Roman, Wyprowadzenie ogólnej postaci kinematyki z uniwersalnym uktadem odniesienia (in Polish). viXra 2017, www.vixra.org/abs/1704.0104. 